Revue internationale P.M.E.

Économie et gestion de la petite et moyenne entreprise

\title{
Les particularités et le fonctionnement des microentreprises informelles à Cuba
}

\section{Zhan Su et Éléa Canipelle}

Volume 10, numéro 1, 1997

URI : https://id.erudit.org/iderudit/1009020ar

DOI : https://doi.org/10.7202/1009020ar

Aller au sommaire du numéro

Éditeur(s)

Presses de l’Université du Québec

ISSN

0776-5436 (imprimé)

1918-9699 (numérique)

Découvrir la revue

Citer cette note

Su, Z. \& Canipelle, É. (1997). Les particularités et le fonctionnement des microentreprises informelles à Cuba. Revue internationale P.M.E., 10(1), 123-141. https://doi.org/10.7202/1009020ar

\section{Résumé de l'article}

Cet article étudie le développement de petites activités économiques privées au Cuba depuis que cet Etat est entré dans une "période spéciale» après l'effondrement de l'Union soviétique. Ces activités se développent en parallèle avec l'économie officielle du pays, formant un secteur informel à la cubaine. Cette recherche exploratoire, qui s'appuie sur la collecte de données sur le terrain, montre que le secteur informel cubain est une économie de survie qui prend place pour pallier l'inefficacité de l'État ou son incapacité à répondre à une forte demande. L'attitude de l'État reste assez ambiguë à l'égard de l'émergence de ce secteur privé. Fonctionnant en microréseaux, et bien que très dynamique, ce secteur n'est pas encore assez structuré. Il semble, cependant, que les activités de survie qui se développent à Cuba forment les entrepreneurs du secteur privé de demain et le futur tissu économique de l'île.
Ce document est protégé par la loi sur le droit d'auteur. L'utilisation des services d’Érudit (y compris la reproduction) est assujettie à sa politique d'utilisation que vous pouvez consulter en ligne.

https://apropos.erudit.org/fr/usagers/politique-dutilisation/ 


\title{
Les particularités et le fonctionnement des microentreprises informelles à Cuba
}

\author{
Zhan SU \\ Éléa CANIPELLE \\ Université Laval
}

MOTS CLÉS
Microentreprise informelle-Entrepreneurs - Cuba-Survie État-Fonctionnement - Comportement

\begin{abstract}
RÉSUMÉ
Cet article étudie le développement de petites activités économiques privées au Cuba depuis que cet État est entré dans une "période spéciale" après l'effondrement de l'Union soviétique. Ces activités se développent en parallèle avec l'économie officielle du pays, formant un secteur informel à la cubaine. Cette recherche exploratoire, qui s'appuie sur la collecte de données sur le terrain, montre que le secteur informel cubain est une économie de survie qui prend place pour pallier l'inefficacité de l'État ou son incapacité à répondre à une forte demande. L'attitude de l'État reste assez ambiguë à l'égard de l'émergence de ce secteur privé. Fonctionnant en microréseaux, et bien que très dynamique, ce secteur n'est pas encore assez structuré. II semble, cependant, que les activités de survie qui se développent à Cuba forment les entrepreneurs du secteur privé de demain et le futur tissu économique de l'île.
\end{abstract}

\section{LES AUTEURS}

Zhan Su est professeur agrégé à la Faculté des sciences de l'administration de l'Université Laval. Ses domaines de recherche portent sur les stratégies d'entreprise, l'entrepreneurship et le management international. Auteur de plusieurs articles dans ces domaines, il agit également comme consultant auprès d'entreprises et d'organisations canadiennes et étrangères.

Éléa Canipelle a complété un M.Sc. en management à l'Université Laval en 1994. Depuis les deux dernières années, elle travaille à Cuba comme consultante pour les entreprises internationales. 


\begin{abstract}
This paper deals with the spread of informal activities in Cuba, since the collapse of the Soviet Union. This so-called "special period", as termed by the government, has not been closely studied to date. This explanatory research intends to increase the level of understanding of these activities, by analysing their functioning and specificities. The methodology that has been used is based on data collection in Cuba. The informal sector appears as an economy of survival in order to bridge the gap between governmental offers and the demands of the population. The attitude of the State towards the development of a private sector remains unclear. The private sector functions with micro-networks for production and distribution. The sector is quite dynamic, however it is not structured enough yet. Finally, activities of survival are training future entrepreneurs and setting up bases for the coming economic pattern.
\end{abstract}

\title{
RESUMEN
}

Ese artículo estudia el desarrollo de las pequeñas actividades económicas privadas a Cuba desde que la Isla ha entrado en un periodo especial después el desmoronamiento del bloque comunista. Esas actividades se desarrollan en paralelo de la economía oficial del país, formando un sector informal a la cubana. La metodología utilisada para esta búsqueda exploratoria se basa en la colecta de dados, por la entrevista semi-directa, la analísis de dados secundarios y la observación directa. Parece ser que el sector informal cubano sea una economía de superviviencia que palia a la ineficacia del Estado ó su incapacidad a contestar a una demanda fuerte. La actitud del Estado queda bastante ambigua enfrente de la emergencia de este sector privado. Funcionando en microredes, y aunque muy dinámico, este sector no es todavía bastante estructurado. Sin embargo, parece ser que las actividades de superviviencia que se desarrollan a Cuba forman los empresarios del sector privado de mañana y la futura organización económica de la isla.

\section{Introduction}

Les événements de 1989, avec l'éclatement de l'Union soviétique et l'effondrement du mur de Berlin, ont entraîné le Cuba dans une «période spéciale», selon l'expression de ses dirigeants, caractérisée par une économie de pénurie, de restriction et de rationnement. L'État n'ayant plus assez de moyens pour subvenir à tous les besoins de la population, les Cubains sont face à leur destin (Habel, 1989; Larifla, 1992). Ainsi, depuis quelques années on assiste, à Cuba, à une explosion de petites activités économiques privées qui, pour la plupart, se développent en parallèle de l'économie officielle du pays, formant ainsi un secteur informel à la cubaine. Situation inédite et riche d'enseignement, le secteur informel cubain et ses acteurs n'ont pourtant pas encore fait l'objet d'études sérieuses. Il est vrai que le phénomène du secteur informel a été observé dans de nombreux pays du tiers monde et son fonctionnement a déjà fait l'objet de nombreuses analyses (BIT, 1972 ; Sethuraman, 1976, 1981 ; Hugon et al., 1977 ; 
Staubus, 1989 ; Abedian et De Smidt, 1990 ; Arellano et al., 1994). Mais, à bien des égards, le cas du Cuba demeure différent de celui des autres pays. Notre recherche veut donc contribuer à mieux comprendre les mécanismes du secteur informel cubain.

Dans la première section de cette étude, nous tenterons de mettre en évidence le cadre conceptuel et méthodologique de notre recherche. La seconde section sera réservée à l'analyse des caractéristiques du secteur informel cubain. Enfin, nous présenterons les résultats de notre enquête sur les microentreprises informelles cubaines et leurs acteurs dans une troisième section.

\section{Cadre conceptuel et méthodologique de la recherche}

\subsection{Secteur informel}

Objet de nombreuses recherches depuis près de vingt-cinq ans, le secteur informel ainsi que ses principaux acteurs, les microentreprises informelles, sont reconnus aujourd'hui comme un phénomène de grande importance dans le monde. De nombreux critères, suivant l'une ou l'autre orientation des chercheurs, ont été avancés pour définir et décrire le secteur informel (BIT, 1972; Hugon et al., 1977 ; Charmes, 1987 ; Rauch, 1991). Selon Ginsburgh et Pestieau (1987), il y a plusieurs façons de distinguer une activité formelle d'une activité informelle. Il peut être question du revenu (déclaré ou non) qu'elle procure, ou du caractère de la production qui lui est associée (contrôlée ou non par l'État), ou encore du type d'échanges (officiels ou non) de biens et de services. L'auteur ajoute plusieurs autres éléments permettant cette distinction. Quant à Verna (1989), il utilise les deux dimensions légalité et légitimité pour définir les activités du secteur informel. Pour Su (1993), les activités du secteur informel, tout en étant illégales, ne sont pas pour autant antisociales. En effet, il est généralement admis que le secteur informel représente un ensemble d'activités économiques marchandes qui échappe au contrôle de l'État en matière d'enregistrement, d'imposition et de toute autre règle officielle, mais joue un rôle positif dans le fonctionnement économique et dans la régulation sociale.

Au demeurant, le secteur informel est devenu aujourd'hui un phénomène dont l'étendue est considérable dans l'économie mondiale. Dans plusieurs pays africains, il constitue le débouché occupationnel le plus important (Ntoula, 1989 ; BIT, 1989 ; Morris et Pitt, 1995). Dans les pays latino-américains les plus touchés par des crises économiques, la majeure partie de la population travaille dans ce secteur, fournissant parfois plus de $40 \%$ du produit intérieur brut (PIB) [De Soto et al., 1986 ; Lopez-Castano, 1987 ; De Miras, 1990]. Par ailleurs, l'économie informelle est estimée à $20 \%$ du PIB en Inde. 
En outre, plusieurs raisons peuvent expliquer l'apparition du secteur informel, et ses mécanismes diffèrent d'un pays à l'autre. D'après des recherches déjà réalisées en Afrique, en Amérique latine et aussi dans certains pays asiatiques, trois grands ensembles d'explications semblent pouvoir être dégagés. Le premier réside dans la défaillance de l'économie formelle en matière de création d'emplois et de source de revenus. En effet, bien que durant les deux dernières décennies l'économie de la plupart des pays du tiers monde ait connu une diminution constante de tous les indices, le marasme économique a obligé plusieurs personnes à se créer des emplois précaires, faisant ainsi du secteur informel un véritable substitut à une structure en crise (Weeks, 1975 ; Penouil et Lachaud, 1985 ; De Bandt et Hugon, 1988).

Une seconde source d'explications serait d'ordre institutionnel et juridique, en raison de l'existence de systèmes administratifs trop contraignants ou de lois trop rigides. L'étude remarquable de De Soto (1989) et son équipe sur le secteur informel au Pérou montre que pour pouvoir installer «légalement» une petite usine de textile à Lima, il faut se rendre à de nombreuses reprises dans sept organismes ou ministères différents en 42 semaines, sans oublier les versements de pots-de-vin, afin d'obtenir les 11 permis requis. Cela explique pourquoi les entrepreneurs de ce pays ont choisi, malgré les risques, de se mettre sous le couvert du secteur informel, pour échapper aux nombreuses tracasseries administratives.

Le troisième groupe de facteurs expliquant l'existence du secteur informel est lié aux aspects politiques. Dans une étude réalisée par Su (1993) sur les causes de l'informalisation des petites activités marchandes en Chine, l'auteur montre que la liberté laissée par le gouvernement chinois, pour ce qui est du développement des activités économiques privées, est réduite par l'idéologie politique et la croyance sociale selon lesquelles ces activités «capitalistes » ne sont pas positives pour la société. Ainsi, influencés à la fois par la perspective de profits intéressants et par le mépris social, les nouveaux entrepreneurs privés chinois essaient soit d'agir dans la clandestinité, soit de gagner de l'argent le plus rapidement possible par tous les moyens (légaux et illégaux).

\subsection{Méthodologie de la recherche}

L'objet à étudier demeurant particulièrement complexe, la recherche sur le phénomène du secteur informel représente donc un grand défi sur le plan méthodologique. La difficulté est d'autant plus grande que, dans le cas de Cuba, la situation est fragile et en perpétuelle mutation. En outre, le régime politique en place affecte énormément l'accès à l'information et remet en question la validité de certaines données. 
Dans le cadre de cette recherche qui se veut de nature exploratoire, nous nous sommes appuyés sur trois instruments de collecte de données, à savoir l'entrevue semi-directive, l'analyse de données secondaires et l'observation directe. Deux collectes de données sur le terrain à Cuba ont été effectuées, respectivement en décembre 1994 et de janvier à mai 1995. Nous avons interrogé un total de 32 «entrepreneurs informels » cubains, que nous avons rencontrés individuellement, en moyenne 3 à 4 fois, lors d'entretiens d'une heure environ. Le sujet étant assez délicat, nous avons dû créer des liens de confiance suffisamment forts afin d'obtenir des informations pertinentes. Les données secondaires venant de diverses sources (périodiques, organismes gouvernementaux cubains, experts cubains et étrangers) ont également été recueillies. Au cours de la période de la collecte de données, nous avons, de manière systématique, effectué une analyse quotidienne des informations recueillies durant les différents entretiens et observations effectués. De nombreuses observations directes, soit sur le lieu de travail (informel), soit sur le lieu de transactions ont été réalisées pour « en extraire les faits confirmant que les conséquences anticipées sur le plan du raisonnement logique existent dans la situation réelle» (Mace, 1988).

\section{Caractéristiques du secteur informel cubain}

\subsection{Esquisse d'une définition du secteur informel cubain}

Une réalité bien particulière prévaut à Cuba, en cela que le seul critère de légalité ne permet pas de circonscrire la sphère du secteur informel à Cuba, car, dans ce pays, la frontière entre le légal et l'illégal reste souvent très floue. Certaines activités, officiellement légales, demeurent très contrôlées et réprimées, alors que beaucoup d'autres, officiellement interdites, s'exercent en toute impunité. L'État tolère et même autorise certains individus à mettre en œuvre des pratiques que la loi condamne parallèlement. C'est notamment le cas des activités économiques privées cubaines, considérées par l'autorité tantôt comme légales, tantôt comme illégales, et dont certaines sont permises ou tolérées, tandis que d'autres sont réprimées. Ainsi, les activités économiques privées cubaines oscillent-elles entre le formel et l'informel (voir la figure 1).

\subsection{L'ambiguïté de l'État cubain envers les activités économiques privées}

Le système communiste, par définition, condamne la propriété privée, l'enrichissement personnel, les différences sociales et les inégalités. Mais face à des problèmes économiques chroniques et à l'inefficacité du secteur étatique, le gouvernement cubain a parfois été obligé d'assouplir ses répressions et de réviser 


\section{FIGURE 1}

\section{La nature des activités du secteur informel cubain}

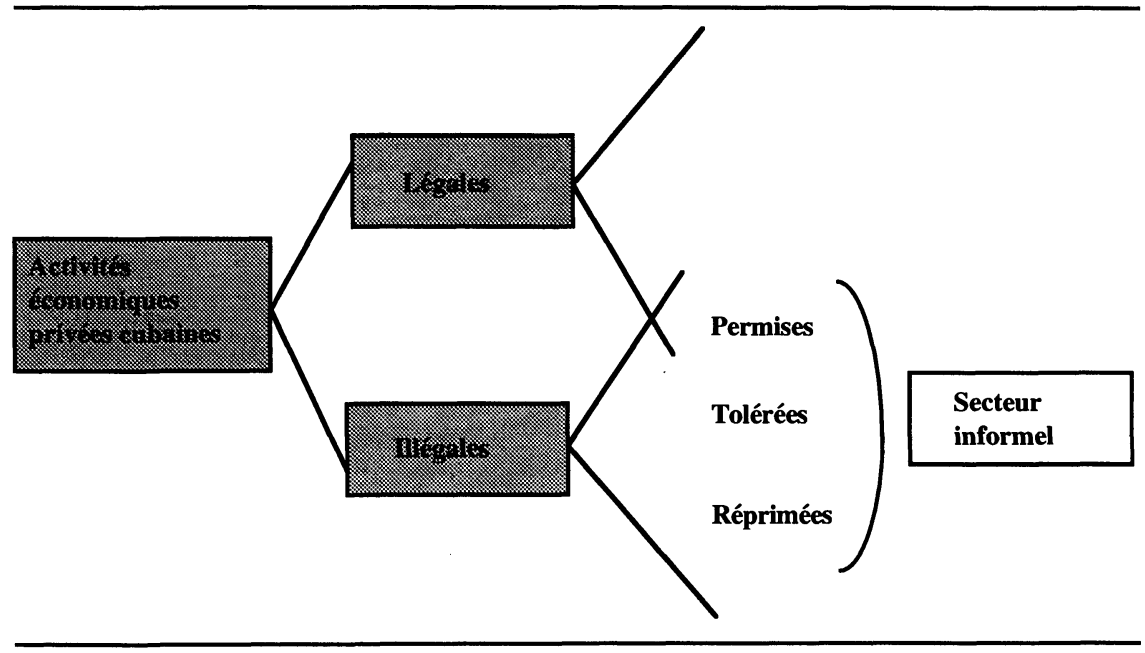

ses positions envers les activités économiques privées. Ainsi, en 1978, l'État avait déjà légalisé quelques activités privées. Mais ces activités ont ensuite été rapidement interdites, car «les résultats ne furent pas concluants » aux yeux du gouvernement. C'est en septembre 1993 que certaines activités privées ont été à nouveau légalisées par la loi 141 de la Constitution cubaine. Parmi celles-ci figurent toutes les petites activités individuelles, telles que les professions de manucure, cireur de chaussure, cordonnier, vendeur de livres, artisans et les marchés libres paysans où les agriculteurs peuvent vendre leur production après avoir pourvu aux besoins de l'État (chaque année, l'État prélève des produits agricoles selon les quotas fixés). Désormais, si l'entrepreneur désire vendre sur le marché officiel, il doit acheter une licence et devra payer un impôt sur le revenu qu'il touche; les taux sont fixés en fonction de la nature du bien ou du service commercialisé et de la quantité vendue.

Mais ce changement ne signifie pas pour autant que le gouvernement s'éloigne de l'idéologie communiste. Le discours du dirigeant cubain est clair sur ce point: «Les réformes conduites ne sont pas fondées sur l'idée de nous éloigner du socialisme, mais sur l'idée de sauver le socialisme» (Castro, 1995). En fait, le travail privé est considéré par l'État comme un mal nécessaire car «si nous [membres du gouvernement] ne pouvons construire de nouvelles usines [faute de moyens] et si nous diminuons les effectifs dans les usines [par nécessité], nous devons trouver une autre source d'emplois, par exemple le travail indépendant; et nul n'ignore ce que signifie le travail indépendant, 
personne n'a de contrôle sur ce type de travail », toujours selon Castro (1995). Le gouvernement cubain se trouvant devant un dilemme, son ambiguité d'attitude et d'actions a, par conséquent, poussé les Cubains à choisir l'évitement du contrôle gouvernemental en travaillant en marge de la légalité officielle, donc, dans le secteur informel.

Le gouvernement adopte actuellement une attitude plutôt tolérante envers le développement du secteur informel à Cuba. Celui-ci le dérange, certes, à cause de ses caractéristiques illégales, mais il lui rend aussi un grand service. En fait, ce secteur informel est, aux yeux du gouvernement, une bouée de sauvetage, un régulateur de tension ou encore une solution à court terme pour se sortir de cette économie de guerre où le rationnement dû à la pénurie de produits de consommation existe, même s'il se garde bien de lui accorder officiellement une place plus importante dans l'économie. La figure 2 explique la politique du gouvernement cubain concernant le développement du secteur informel.

\section{Figure 2}

\section{La double attitude de l'État envers les activités informelles}

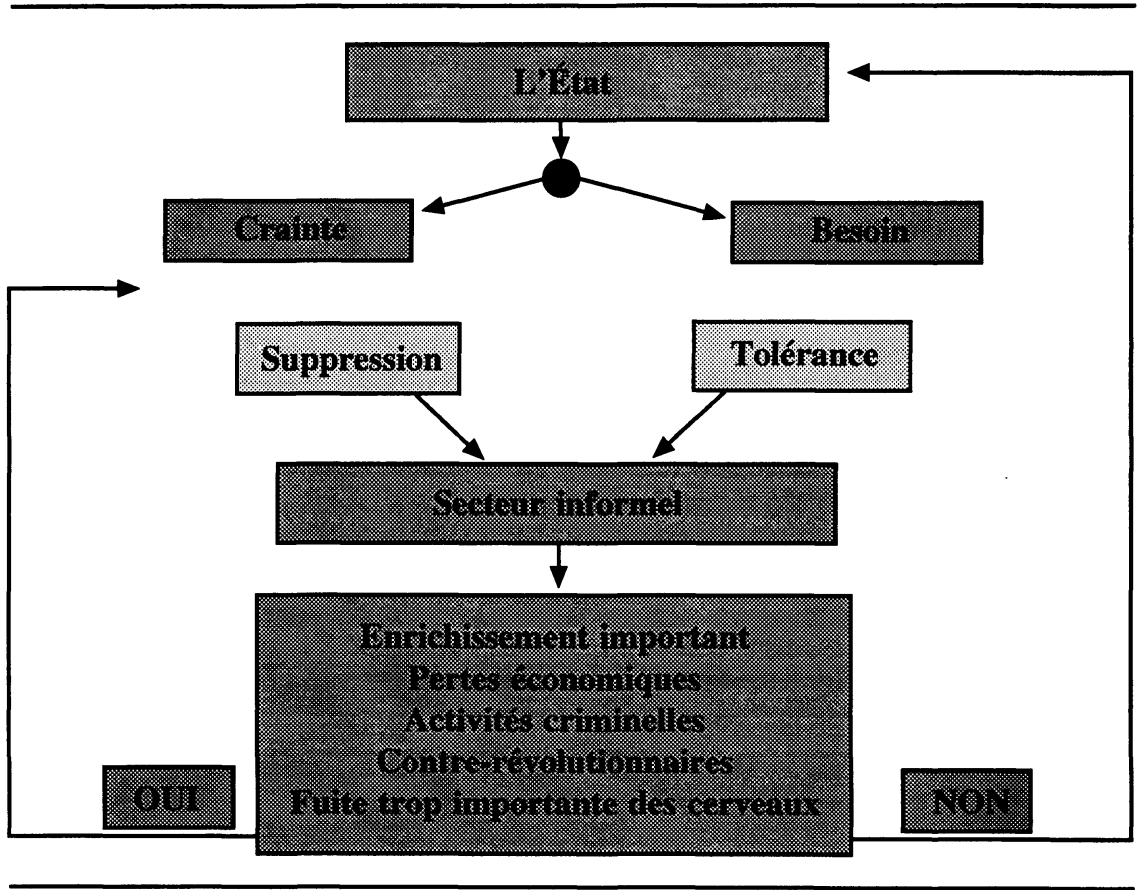


En somme, l'État accepte le développement d'activités informelles dans des secteurs où il n'est pas lui-même en mesure de répondre à la demande. En effet, dans de nombreux secteurs vitaux de l'économie, l'État est incapable de proposer, à une vaste clientèle, un service de qualité à des prix raisonnables. Par conséquent, il laisse se développer un marché noir de produits alimentaires, de produits pour l'hygiène corporelle, de services essentiels (plomberie, électricité, maçonnerie, etc.). Dans des secteurs comme l'habillement (textile, chaussure), l'État cherche à se désengager, car ces activités ne sont plus suffisamment rentables. L'économie souterraine tolérée par l'État ne lui fait donc aucune concurrence directe. Bien au contraire, elle le soulage en organisant des secteurs d'activités jusqu'ici paralysés. La mauvaise organisation de ces activités et la gestion « socialiste» condamnaient leur rentabilité et leur efficacité, mais leur nécessité justifie pourtant le développement d'un secteur privé, fût-il clandestin, capable de répondre aux attentes des consommateurs. Cependant, l'État peut devenir intolérant à l'égard des activités informelles lorsqu'il risque de ne plus contrôler la situation, du fait d'un enrichissement trop marqué de certaines personnes, de pertes économiques trop importantes, de développement d'activités criminelles (comme le trafic de drogue), de critiques contre le régime et de fuite des cerveaux.

\subsection{Le secteur informel: une économie de subsistance}

La «période spéciale » est tellement difficile que la population cubaine n'a d'autre choix que de s'approvisionner sur le marché noir aujourd'hui. Les spécialistes que nous avons rencontrés estiment qu'environ $85 \%$ de la population cubaine consomme, d'une manière ou d'une autre, les services du secteur informel. Les magasins d'État étant vides, la population se tourne vers un marché en mesure de satisfaire ses besoins. Depuis 1989, le secteur informel ne cesse de se développer, attirant de nouveaux entrepreneurs. Aujourd'hui, environ les deux tiers de la population active se sont impliqués dans des activités informelles. Dans un pays en transition comme Cuba, il est plus facile et plus rémunérateur de travailler dans l'économie parallèle. D'ailleurs, selon les résultats de notre enquête, il ressort que pour ceux qui travaillent régulièrement dans des activités informelles, leur gain pourrait représenter jusqu'à $99 \%$ de leur revenu total. Faute d'informations et de statistiques, il est impossible de définir avec exactitude la part réelle du secteur informel dans l'économie cubaine. Il apparaît cependant que, dans des secteurs tels que l'industrie légère et les services, sa part est extrêmement importante.

L'économie informelle de Cuba reste avant tout une économie de subsistance. Notre enquête sur le terrain permet de constater qu'en période de crise économique et sociale, la décision d'opérer sur le secteur informel est 
essentiellement motivée par le désir de survivre. Le goût de l'initiative et du métier - plus rarement celui du risque - constituent aussi des facteurs de motivation pour l'entrepreneur du secteur informel. Par ailleurs, la relative tolérance de l'État à l'égard de l'économie parallèle et la multiplication des activités informelles sécurisent les entrepreneurs déjà actifs et tendent à convaincre les entrepreneurs en devenir.

\subsection{Une typologie des activités informelles cubaines}

Dans le but de présenter de façon précise les activités du secteur informel cubain, nous avons développé une grille de classification de ces activités. Trois types d'activités pourraient être identifiés, les voici.

\subsubsection{Les activités de production ou de création}

L'acteur fabrique le produit à l'aide de matériaux qu'il a fabriqués, achetés ou volés et qu'il devra vendre. Nous avons regroupé les types d'activités en fonction de la matière première utilisée. Voici quelques exemples : bois (fabricant de mobilier); pierre ou minerai (utilisation du marbre); matériaux de construction (construction de maison ou de pièces supplémentaires); divers (la création d'artisanat); produits agricoles (tout ce qui est récolté par le paysan); élevage (pour la transformation en viande). La fabrication n'est pas en elle-même nécessairement une activité informelle. En revanche, elle le devient lorsque la vente des produits fabriqués se fait de façon informelle ou lorsque les matières premières ont été obtenues illégalement.

\subsubsection{Les activités de revente}

L'acteur ne fait que vendre le produit qui lui appartient, qu'il a acheté ou parfois volé. La distribution de différents types d'activités pourrait se faire, à la rigueur, en fonction du produit vendu (produits frais, matières premières, produits pharmaceutiques, œuvres d'art, produits de luxe, tabac, alcool, pièces détachées, essence, produits électroniques, contrefaçon : le plus souvent de tabac ou de rhum).

\subsubsection{Les activités de services}

L'acteur offre ses services à domicile ou dans la rue.

Dans le cadre de notre étude sur le terrain, nous avons été mis en présence d'un total de 35 types d'activités, répertoriés dans le tableau 1. 
TABleau 1

Les activités informelles relevées à Cuba

\begin{tabular}{lll}
\hline \multicolumn{1}{c}{ Production } & \multicolumn{1}{c}{ Revente } & \multicolumn{1}{c}{ Services } \\
\hline Fabrication à base de : & - Objets d'art & - Femme de ménage \\
- Bois & - Matières premières & - Maître d'hôtel \\
- Métaux & - Produits pharmaceutiques & - Prostituée \\
- Matériaux de construction & - Tabac, alcool, essence & - Médecin \\
- Cuir & - Électronique, Hi-fi & - Coiffeur \\
- Produits agricoles & - Produits frais & - Professeur \\
- Viande (élevage) & - Pièces détachées & - Vendeur de pesos \\
- Produits divers & - Contrefaçon & - Esthéticienne \\
- Pierres ou minerais & - Vente d'appartements & - Électricien \\
& & - Jardinier \\
& & - Coiffeur \\
& & - Couturière \\
& & - Plombier \\
& & - Garagiste \\
& & - Restaurateur \\
& & - É̂telier " \\
& & - Loueur d'un journal dissident \\
& & $\mathbf{1 8}$ \\
\hline Total par activité: 8 & & Total = 35 activités \\
\hline
\end{tabular}

\section{Les microentreprises informelles cubaines et leurs acteurs selon l'enquête}

\subsection{Un aperçu des résultats de l'enquête}

Le tableau 2 donne un aperçu des microentreprises informelles cubaines ainsi que de ses acteurs étudiés sur le terrain.

\subsection{Les caractéristiques des microentreprises informelles cubaines}

\subsubsection{Caractéristiques générales}

Les activités observées s'exercent dans trois catégories d'entreprises. Les plus fréquentes sont les entreprises de services (pour $51 \%$ ), viennent ensuite les entreprises de revente ( $26 \%$ ) puis de transformation (ou fabrication) [ $23 \%$ ]. La taille de l'entreprise varie très peu et tourne autour d'une à quatre personnes. 


\section{TABLEAU 2}

\section{Données recueillies lors de nos entretiens avec 32 entrepreneurs du secteur informel}

\begin{tabular}{|c|c|c|c|}
\hline Questions posées & Producteurs & Revendeurs & Services \\
\hline $\begin{array}{l}\text { Combien de représentants } \\
\text { de chaque secteur ont été } \\
\text { interrogés? }\end{array}$ & 6 & 11 & 15 \\
\hline $\begin{array}{l}\text { Quel niveau scolaire } \\
\text { avez-vous? }\end{array}$ & $\begin{array}{l}\text { Secondaire : } 3 \\
\text { Universitaire : } 2 \\
\text { Spécialité : } 1\end{array}$ & $\begin{array}{l}6 \\
3 \\
2\end{array}$ & $\begin{array}{l}4 \\
5 \\
6\end{array}$ \\
\hline $\begin{array}{l}\text { Avez-vous un emploi } \\
\text { officiel? }\end{array}$ & Oui 4 ; Non 2 & Oui 6 ; Non 5 & Oui 9 ; Non 6 \\
\hline $\begin{array}{l}\text { Combien d'emplois } \\
\text { officiels avez-vous? }\end{array}$ & Officiel : 1 & Officiel : 1 & Officiel : 1 \\
\hline $\begin{array}{l}\text { Combien gagnez-vous } \\
\text { officiellement? }\end{array}$ & Entre 200 et 300 pesos & 200 à 350 pesos & Entre 120 et 500 pesos \\
\hline $\begin{array}{l}\text { Combien avez-vous } \\
\text { d'employés ou } \\
\text { de complices pour } \\
\text { votre activité informelle? }\end{array}$ & $\begin{array}{l}\text { Employés : } 2 \text { maximum } \\
\text { Complices : } 2-4\end{array}$ & $\begin{array}{l}\text { Employés : } 0 \\
\text { Complices : } 2-6\end{array}$ & $\begin{array}{l}\text { Employés: } 0-4 \\
\text { Complices : } 0-3\end{array}$ \\
\hline $\begin{array}{l}\text { Combien de temps } \\
\text { consacrez-vous par jour } \\
\text { à l'activité? }\end{array}$ & $\begin{array}{l}\text { Avec E* : } 6 \mathrm{~h} \\
\text { Sans E. : Le maximum }\end{array}$ & $\begin{array}{l}\text { Avec E. : } 5 \mathrm{~h} \\
\text { Sans E.: Tout le jour }\end{array}$ & $\begin{array}{l}\text { Avec E. : } 5 \mathrm{~h} \\
\text { Sans E.: 6-7 h }\end{array}$ \\
\hline $\begin{array}{l}\text { Où exercez-vous } \\
\text { votre activité : } \\
\text { Maison, Client, Rue? }\end{array}$ & $\begin{array}{l}2 \text { Maison } \\
1 \text { Client } \\
3 \text { Rue }\end{array}$ & $\begin{array}{l}4 \text { Maison } \\
6 \text { Client } \\
1 \text { au travail }\end{array}$ & $\begin{array}{l}6 \text { Maison } \\
9 \text { Client }\end{array}$ \\
\hline $\begin{array}{l}\text { Pensez-vous à fidéliser } \\
\text { votre clientèle? }\end{array}$ & Oui 5 ; Non 1 & Oui 11 & Oui 15 \\
\hline $\begin{array}{l}\text { Quels moyens utilisez-vous } \\
\text { pour faire connaître } \\
\text { votre activité? }\end{array}$ & $\begin{array}{l}\text { Démarchage : } 4 \\
\text { Bouche à oreille : } 2 \\
\text { Carte de visite : } 0 \\
\text { Hasard : } 0\end{array}$ & $\begin{array}{l}3 \\
4 \\
1 \\
3\end{array}$ & $\begin{array}{l}6 \\
6 \\
0 \\
3\end{array}$ \\
\hline $\begin{array}{l}\text { D'où proviennent } \\
\text { les matières premières } \\
\text { et/ou le produit? }\end{array}$ & $\begin{array}{l}\text { Volées : } 4 \\
\text { Achetées : } 1 \\
\text { Produites : } 1\end{array}$ & $\begin{array}{l}4 \\
6 \\
1\end{array}$ & 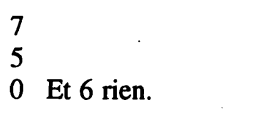 \\
\hline $\begin{array}{l}\text { Investissez-vous réguliè- } \\
\text { rement ou votre investis- } \\
\text { sement de base suffit-il? }\end{array}$ & $\begin{array}{l}2 \text { Aucun } \\
1 \text { De base } \\
3 \text { Régulièrement }\end{array}$ & $\begin{array}{l}6 \text { Aucun } \\
4 \text { De base } \\
1 \text { Régulièrement }\end{array}$ & $\begin{array}{l}8 \text { Aucun } \\
2 \text { De base } \\
5 \text { Régulièrement }\end{array}$ \\
\hline $\begin{array}{l}\text { Combien représentent } \\
\text { vos bénéfices par rapport } \\
\text { à vos revenus officiels? }\end{array}$ & $99 \%$ & $99 \%$ & $99 \%$ \\
\hline Vos profits sont-ils stables? & Oui $2 ; \quad$ Non 4. & Oui $5 ; \quad$ Non 6. & Oui $10 ;$ Non 5. \\
\hline $\begin{array}{l}\text { Avez-vous peur des } \\
\text { représailles de l'État? }\end{array}$ & Oui 1; Non 5. & Oui $2 ; \quad$ Non 9. & $\begin{array}{l}\text { Oui } 1 ; \text { Non } 11 ; \\
\text { Ne sait pas } 3 \text {. }\end{array}$ \\
\hline $\begin{array}{l}\text { La police est-elle au } \\
\text { courant de votre activité? }\end{array}$ & $\begin{array}{l}\text { Oui } 3 \text {; } \\
\text { Ne sait pas } 3 \text {. }\end{array}$ & $\begin{array}{l}\text { Oui } 9 \text {; } \\
\text { Ne sait pas } 2 \text {. }\end{array}$ & $\begin{array}{l}\text { Oui } 14 \text {; } \\
\text { Ne sait pas } 1 \text {. }\end{array}$ \\
\hline
\end{tabular}

* E : emploi officiel 
L'entreprise peut être installée soit chez l'entrepreneur même, et dans ce cas, elle est dite fixe (38\%), soit chez son client, elle est alors semi-ambulante (50\%), soit dans la rue, et est ainsi ambulante $(8 \%)$. Il reste aussi une autre possibilité : l'entrepreneur utilise le lieu de son travail (4\%). Selon les spécialistes de Cuba, les résultats obtenus semblent assez représentatifs de la réalité du secteur informel de l'île. Nous avons constaté que le degré de spécialisation des entreprises variait selon les circonstances opportunes rencontrées par l'entrepreneur. Dans certains cas, l'acteur n'a pas de domaine précis d'activité et est prêt à exercer toutes sortes d'activités, il est donc polyvalent. Mais de façon générale, une fois que «l'entreprise » est lancée et organisée, l'acteur aura plutôt tendance à se spécialiser dans son champ d'activités et tentera d'améliorer le fonctionnement de son entreprise.

\subsubsection{Le profil des acteurs}

L'acteur agit souvent seul : la majorité des entreprises sont individuelles ou encore familiales, rarement associatives. Pour la majorité des acteurs, l'activité du secteur informel est un travail à temps partiel dans le sens où ils ont besoin de leur travail officiel pour obtenir leur matière première. En revanche, l'activité du secteur informel les accapare beaucoup. En moyenne, l'entrepreneur qui a deux emplois (60\% des entrepreneurs interrogés) consacrera de cinq à sept heures par jour à son activité informelle. Certains autres considèrent l'activité informelle comme un travail à temps plein.

Les débuts du secteur informel ont vu fleurir des activités conduites par des individus pas nécessairement instruits, mais «débrouillards». Mais aujourd'hui, les jeunes ayant un niveau d'instruction élevé préfèrent souvent délaisser leur profession pour travailler au noir dans une activité plus rentable et surtout payée en dollars américains. Dans notre échantillon, ils ont tous au moins l'équivalent d'un niveau secondaire, tandis que $31 \%$ détiennent un diplôme universitaire et $28 \%$ une spécialisation (infirmière, électronicien, etc.). Ainsi, nous constatons que des chirurgiens, des mathématiciens, des philosophes et bien d'autres s'improvisent vendeurs de cigares, restaurateurs, « hôteliers », etc., dans une nouvelle économie où le degré de qualification n'est pas un critère de réussite. De formations diverses, les nouveaux entrepreneurs apprennent vite les quelques rudiments du commerce nécessaires pour se lancer. Puis, d'expérience en expérience, ils affinent leurs méthodes de fonctionnement. Les façons de faire ne varient pas trop d'un secteur à un autre puisque tous les entrepreneurs font face aux mêmes problèmes d'approvisionnement, de production et de vente dans un environnement où les canaux officiels ne le permettent pas. 


\subsubsection{L'attitude envers l'autorité}

L'environnement cubain est, par définition, hostile au développement d'activités de ce type, mais la réalité est plus complexe. Les activités informelles étant illégales, même si tolérées, en cas de problème, l'entreprise est persécutée et les acteurs sont condamnés. Pourtant, peu d'entrepreneurs (12\%) ont réellement peur des représailles de l'État ; $7 \%$ n'ont aucune crainte et $9 \%$ restent sans opinion. D'ailleurs, aucun acteur interrogé ne pense être inquiété par le gouvernement, car, selon eux, leurs activités ne sont pas encore suffisamment développées ni suffisamment rentables pour éveiller des soupçons. Souvent, les fonctionnaires et les représentants de l'ordre public ont connaissance de ces activités et en deviennent indirectement les complices silencieux.

\subsubsection{La gestion des activités}

Les activités informelles ont obligatoirement besoin, pour fonctionner, de l'appui de la population. Des activités de nature plutôt antisociale ont du mal à se développer faute de clients. Les entrepreneurs rencontrés semblent en être bien conscients et tentent de travailler dans ce sens. La qualité des produits vendus est globalement satisfaisante, car l'acteur cherche à fidéliser sa clientèle, et $97 \%$ des entrepreneurs interrogés tentent de faire de même en vendant de la qualité à prix abordable et en satisfaisant le plus souvent possible la demande. L'acteur tente d'être à l'écoute des besoins de sa clientèle. Ayant peu de moyens, il ne peut se permettre de faire de grandes campagnes publicitaires pour attirer le client. Ainsi, beaucoup se fient au démarchage $(41 \%)$ ou au bouche à oreille $(37 \%)$ et, plus rarement, au hasard (19\%) et à la distribution de cartes de visite (3\%).

\subsubsection{Le financement des activités}

Sur le plan financier, les entreprises sont encore trop modestes pour nécessiter un financement extérieur. Généralement, elles arrivent rapidement à l'autofinancement. Seulement $28 \%$ des acteurs interrogés investissent régulièrement dans leur entreprise ; $22 \%$ investissent une fois au lancement de leur activité et $50 \%$ disent ne pas avoir à investir. Il est vrai que l'économie parallèle étant définie comme une économie avant tout illégale, les entrepreneurs n'ont ni l'envie ni les moyens de consacrer une partie de leur budget personnel à leur entreprise.

Par ailleurs, l'activité informelle à Cuba ne requiert souvent que très peu d'équipement. L'acteur possède, au mieux, quelques instruments très simples, nécessaires à l'exercice de son métier. De même, les techniques utilisées sont rudimentaires, restent très traditionnelles et sont parfois obsolètes, mais encore suffisantes pour répondre à la demande. Notre étude a révélé que l'investissement en machinerie est minime, pour ne pas dire inexistant. La fabrication 
est donc majoritairement artisanale et ne permet pas de produire en grande quantité. En revanche, il existe une fabrication plus structurée, mais encore relativement peu développée : celle de la contrefaçon. Lors de notre recherche, nous avons observé deux sortes de contrefaçons : celle des cigares et celle du rhum.

\subsubsection{L'approvisionnement}

Dans une économie de pénurie, l'approvisionnement est un problème majeur pour l'ensemble de la population et, plus particulièrement, pour les entrepreneurs. Chaque acteur tente de trouver des sources d'approvisionnement fiables et peu coûteuses, qui sont la production personnelle, l'achat et même le vol. Dans notre échantillon, la première source d'approvisionnement utilisée est le vol de l'État ou du particulier (47\% des acteurs de l'ombre s'approvisionnent par cette voie, méthode qui devient de plus en plus prisée tant la police est indulgente). La deuxième est la production personnelle qui concerne plus particulièrement les produits agricoles. Peu nombreux sont les entrepreneurs qui utilisent ce type d'approvisionnement $(6 \%)$, qui se révèle long et plus coûteux. La troisième source concerne des acteurs possédant déjà le produit ou la matière première. Les anciens bourgeois qui détiennent des biens tels que de la vaisselle, des meubles, de l'argenterie, etc., les vendent progressivement au marché noir. La quatrième est la revente de matière première achetée à prix modique comme celle de la libreta. En effet, les produits proposés à des prix dérisoires grâce aux carnets de rationnement sont souvent revendus à des prix plus élevés sur le secteur informel.

Remarquons que les caractéristiques substantielles du secteur informel de Cuba, exposées ici, montrent à quel point l'économie parallèle est embryonnaire. Il s'agit davantage de plusieurs activités désorganisées se développant anarchiquement dans l'ombre que d'un marché informel structuré.

\subsection{Le fonctionnement des microréseaux dans le secteur informel}

Dans le secteur informel cubain, nous avons constaté deux grands types de réseaux complémentaires : le réseau de la production et le réseau de la distribution (voir la figure 3). Toutefois, le terme réseau doit être employé avec nuance, car le secteur informel cubain est éclaté. Il ne s'agit pas véritablement d'une organisation monolithique ni d'une « organisation clandestine formée par un certain nombre de personnes en relation directe ou indirecte les unes avec les autres et obéissant aux mêmes directives » (définition simple du mot réseau issu du petit Robert). Dans le cas de Cuba, l'économie parallèle est formée de nombreux « microréseaux » qui ne sont pas interreliés entre eux. On ne peut pas vraiment parler d'organisation du secteur informel, mais plutôt d'une improvisation des 
FIGURE 3

Le fonctionnement des activités informelles en réseau

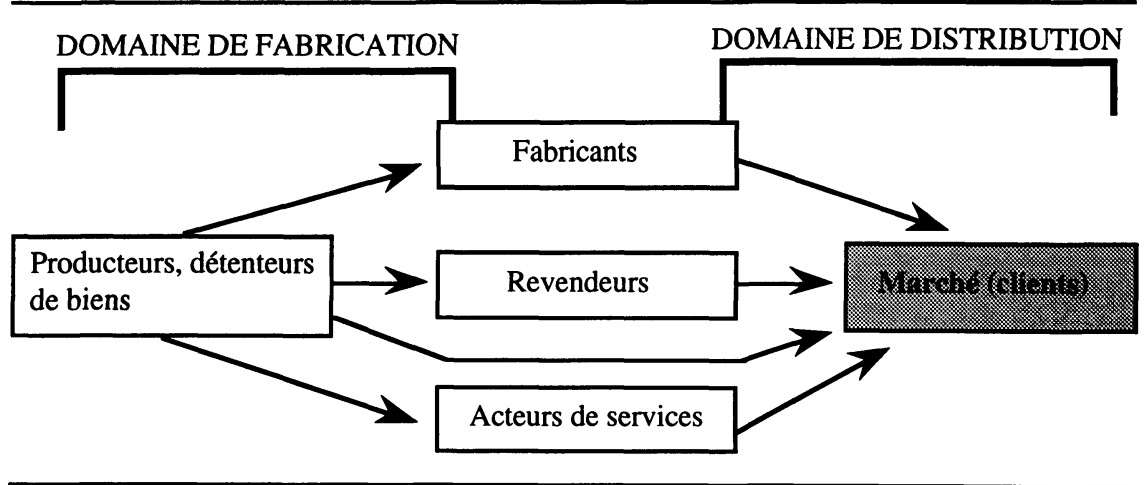

activités informelles. L'objectif de l'entrepreneur cubain est de gagner le plus d'argent possible et, donc, d'essayer de vendre beaucoup. Pour ce faire, chaque acteur tente d'élargir au maximum son réseau de production et de distribution.

\subsubsection{Les scénarios du réseau de la production}

- Le producteur vend directement sa matière première à un fabricant qui la transforme en produit fini destiné à la vente directe ou indirecte (en ayant recours aux services d'un revendeur). Un éleveur de la province de la Havane disait récupérer les peaux de ses animaux une fois abattus, dans le but de les vendre à des fabricants d'articles de cuir (sacs, ceintures, chaussures, etc.). La matière première est ainsi travaillée pour fabriquer un article revendable sur les marchés de l'économie parallèle.

- Le producteur peut également vendre sa matière première aux acteurs du secteur des services. Prenons l'exemple le plus souvent rencontré : la restauration. Isabelle, gérante d'un palladar, obtient toutes ses provisions de petits producteurs agricoles qui viennent lui livrer fruits, légumes, viandes, etc.

- Ceux que nous appelons les détenteurs correspondent aux personnes possédant des biens (mobiliers ou immobiliers) à vendre. Une bonne partie de l'ancienne bourgeoisie cubaine a dû vendre petit à petit son patrimoine pour conserver un niveau de vie décent. $D$ 'autres détenteurs de biens se les ont procurés de façon illégale, par le vol, qui devient un moyen d'approvisionnement de plus en plus prisé. En fonction des occasions qui se présentent, le voleur revendra de la matière première aux fabricants (nous faisons, ici, allusion à un ouvrier qui construit des 
immeubles dans le quartier de Miramar, à la Havane, et qui pour « arrondir les fins de mois » subtilise du matériel de construction - produit très prisé sur le marché noir - pour la revente). Le vol de produits finis (appareils photo, chaînes hi-fi, bijoux, etc.) est malheureusement en expansion. Les biens volés aux touristes étrangers et aux résidents cubains sont ainsi vendus sur le marché noir par l'intermédiaire de revendeurs et de travailleurs du secteur des services (p. ex : la décoration de la salle à manger d'un palladar a été réalisée avec du matériel volé sur le chantier de construction d'un hôtel).

\subsubsection{L'étape cruciale de la distribution}

La deuxième partie du maillage est une étape cruciale puisqu'il s'agit de la vente. La figure 3 montre que le client a plusieurs possibilités pour s'approvisionner :

- faire affaire directement avec le fabricant parce qu'il connaît l'acteur et son produit (Nous pensons ici au fabricant de poupées géantes qui n'a jamais fait appel à un intermédiaire pour vendre son produit et qui désormais a une clientèle directe grandissante.);

- passer par un revendeur; dans ce cas, il paie un peu plus cher son produit ;

- acheter au receleur, lequel propose appareils photo, montres, bijoux, jeans, etc., à des prix défiant toute concurrence ;

- faire appel au secteur des services pour, par exemple, faire réparer le moteur de sa voiture.

Dans l'ensemble, nous pouvons répertorier trois logiques de vente: la vente organisée, la vente «sauvage » et la vente de prestations de services.

\section{La vente organisée}

On constate que, contrairement à ce que laisse supposer le premier contact avec le marché noir, le vendeur n'est souvent pas seul à exercer son activité. Il est épaulé par plusieurs acolytes, qui sont généralement le producteur ou le distributeur, et parfois même un agent de l'État qui, en échange d'une commission, ferme les yeux sur ses activités. Ce fonctionnement en réseau s'étend presque à l'ensemble des activités de fabrication et de revente.

De prime abord, il semble que ce soit autour du producteur de l'objet vendu que se développe l'activité illégale. Détenteur du produit, le fabricant pourrait être, en effet, l'initiateur de l'activité. Or, l'expérience montre que le vendeur est le maillon le plus important de la chaîne. Il connaît de multiples clients et leurs attentes; il a leur confiance et se trouve, de surcroît, en relation avec plusieurs producteurs ou revendeurs. Son réseau d'informations et de 
connaissances n'a pas de prix ; par conséquent, il le fait payer cher. L'individu qui revend des objets d'art (tableaux, meubles, bijoux, etc.) dont les propriétaires souhaitent se débarrasser agit comme courtier et fait valoir un carnet d'adresses bien garni.

\section{La vente "sauvage»}

Ici, nul besoin d'un carnet d'adresses pour réussir : il s'agit de la vente «sauvage » dans la rue. Pour l'exercice de ces activités, l'acteur ne s'adresse plus à une clientèle fidèle, mais plutôt à une clientèle de passage (touristes). Ainsi, des « courtiers » passent des jours entiers à arpenter les rues des villes comme La Havane, Santiago, Cienfuegos ou encore Pinar del Rio afin de trouver des acheteurs potentiels de divers produits (cigares, alcool, bicyclettes, etc.). Ils doivent s'assurer de pouvoir répondre rapidement à la demande en ayant établi au préalable un réseau dense de contacts auprès de producteurs. Il en est autrement pour les activités de services.

\section{La vente de prestations de services}

Le fonctionnement des activités du secteur des services présente d'autres caractéristiques. Afin de travailler clandestinement comme maître d'hôtel, jardinier, médecin, etc., l'acteur n'a pas besoin de se constituer un réseau de connaissances aussi développé que dans le cas des activités de revente et de production. Les acteurs font plutôt du démarchage auprès des personnes susceptibles d'avoir besoin de leurs services. L'essor de leur activité repose davantage sur le hasard et l'intuition de l'entrepreneur que sur l'exploitation d'un réseau bien établi.

\section{Conclusion}

Le secteur informel cubain est en constant développement et rend actuellement un grand service à un nombre important de consommateurs, palliant l'inefficacité de l'État ou son incapacité à répondre à une forte demande. L'attitude de l'État reste, cependant, assez ambiguë à l'égard de l'émergence de ce secteur privé. Les mesures prises par le gouvernement autorisant le développement de certaines activités privées augurent d'une ouverture possible de l'économie. Cependant, dans l'ensemble, l'environnement reste hostile aux entrepreneurs privés cubains, car, comme le déclarent les dirigeants cubains : «Les réformes conduites ne sont pas fondées sur l'idée de nous éloigner du socialisme, mais sur l'idée de sauver le socialisme. » (Castro, 1995)

Dans tous les cas, les activités de survie qui se développent à Cuba forment les entrepreneurs du secteur privé de demain grâce à l'expérience qu'ils en tirent. Cependant, il serait intéressant de suivre l'évolution, ou plutôt la création, du secteur privé, d'en étudier les répercussions sur le développement de 
l'économie parallèle et de voir si les activités informelles sont une menace pour le régime actuel et une force pour le régime de demain. Ce secteur, qui se structure, se ramifie de façon tout à fait autonome sous l'impulsion d'entrepreneurs privés créatifs, audacieux, rompus aux méthodes de gestion capitalistes élémentaires, pourrait constituer rapidement une source de revenus importante pour l'État (par l'impôt sur les sociétés que les économistes cubains pensent instituer) et servira sans doute de modèle au développement futur de l'économie de l'île.

\section{Bibliographie}

Abedian, I. et M. DESMidT (1990), «The informal economy in South Africa», The South African Journal of Economics, vol. 58, n 4, p. 404-424.

AREllano, R., Y. GASSE et G. VERNA (1994), Les entreprises informelles dans le monde, Sainte-Foy (Québec), Les Presses de l'Université Laval.

BUREAU INTERNATIONAL DU TRAVAIL (1972), Employment Incomes and Equality: A Strategy for Increasing Productive Employment in Kenya, Genève.

BUREAU INTERNATIONAL DU TRAVAIL (1989), Le rapport sur l'emploi en Afrique 1988, Addis-Abeba, Éthiopie, OIT-PECTA.

CASTro, F. (1995), Discours de Janvier 1995, Documentation du gouvernement cubain.

Charmes, J. (1987), «Débat actuel sur le secteur informel», Revue Tiers Monde, $\mathrm{n}^{0} 112$, p. 855 .

De BANDT, J. et P. Hugon (1988), Les tiers nations en mal d'industrie, Paris, Economica.

DE Miras, C. (1990), «État de l'informel. Informel et État. Illustrations sudaméricaines », Revue Tiers Monde, $\mathrm{n}^{\circ}$ 122, avril-juin, p. 377.

DE SoTo, H. (1989), «When entrepreneurship is illegal : underdevelopment, black markets and revolution in Latin America », Entrepreneurship Forum, New York, hiver.

De Soto, H., E. GHERSI et M. GHIBELINI (1986), El otro sendero: la revoluciòn informal, Lima Editions El Barranco.

DEBLE, I. et P. HUGON (1982), Vivre et survivre dans les villes africaines, Paris, Presses universitaires de France.

Ginsburg, V. et P. Pestieau (1987), L'économie informelle, Paris, Édition Labor.

HABEL, J. (1989), Rupture à Cuba: Le castrisme en crise, Paris, La Brèche.

Hugon, P., N.L. ABADIE, et A. MoRICE (1977), La petite production marchande et l'emploi dans le secteur informel: le cas africain, Paris, Université de Paris I. 
LARIFLA, L. (1992), «Cuba: Crise du système politico-économique et recherche de nouvelles voies ", Le Courrier des Pays de l'Est, no 372, p. 47-64.

LOPEZ-CASTANOS, H. (1987), «Secteur informel et société moderne : l'expérience colombienne », Revue Tiers Monde, vol. 28, n 110, p. 369-380.

MACE, G. (1988), Guide d'élaboration d'un projet de recherche, Sainte-Foy (Québec), Les Presses de l'Université Laval.

MORRIS, M.H. et L.F. PITT (1995), «Informal sector activity as entrepreneurship: insights from a South African township », Journal of Small Business Management, vol. $33, \mathrm{n}^{\circ} 1$, p. $78-86$.

NTOUla, R. (1989), "Spaza shops : backbone of the informal sector », African Business \& Chamber of Commerce Review, vol. 16, $\mathrm{n}^{0}$ 6, p. 5-10.

PENOUIL, M. et M. LACHAUd (1985), Le développement spontané. Les activités informelles en Afrique, Paris, Édition A. Pedone.

RAUCH, J.E. (1991), « Modelling the informal sector formally », Journal of Development Economics, vol. 35, $\mathrm{n}^{\circ} 1$, p. 33-47.

Sethuraman, S.V. (1976), «Le secteur urbain non structuré, concept, mesure en action », Revue Internationale du Travail, vol. 114, n 1, p. 79-92.

SethuRaman, S.V. (1981), The Urban Informal Sector in Developing Countries: Employment, Poverty, and Environment, Genève, Bureau international du travail.

Staubus, J. (1989), «Latin America's black economies », Multinational Business, vol. $4, n^{0} 4$, p. $35-39$.

SU, Z. (1993) «Essai sur les causes de l' “ informalisation" de petites activités marchandes en Chine », Revue Tiers Monde, vol. 34, $\mathrm{n}^{0}$ 135, p. 672-685.

VERnA, G. (1989), «Légalité ou légitimité : les pièges du tiers monde », Document de travail, Sainte-Foy (Québec), Faculté des sciences de l'administration de l’Université Laval.

WEEKS, J. (1975), «L'extension de l'emploi dans le secteur urbain non structuré des pays en voie de développement », Revue Internationale du Travail, vol. 111, janvier-juin, p. 1-14. 